\title{
Complex Unit Roots and Business Cycles: Are They Real?
}

\author{
Herman J . Bierens \\ Pennsylvania State University, USA, and Tilburg University
}

September 28, 1999

\begin{abstract}
A bstract
In this paper the asymptotic properties of ARMA processes with complex-conjugate unit roots in the AR lag polynomial are studied. These processes behave quite dixerently from regular unit root processes (with a single root equal to 1 ). In particular, the asymptotic properties of a standardized version of the periodogram for such processes are analyzed, and a nonparametric test of the complex unit root hypothesis against the stationarity hypothesis is derived. This test is applied to the annual change of the monthly number of unemployed in the US, in order to see whether this time series has complex unit roots in the business cycle frequencies.
\end{abstract}

\section{Introduction}

The current literature on non-seasonal unit root processes focuses almost entirely on the case of real unit roots (all equal to 1 ). A notable exception is the recent work by Gregoir (1999a,b,c). In the ..rst two papers, Gregoir studies covariance stationary vector moving average (VMA) processes where the determinant of the lag polynomial matrix involved has multiple real and/ or complex unit roots. In the third paper, Gregoir derives a parametric test for a pair of complex conjugate unit roots in an AR(2) process with white noise errors. In this paper, however, we will take a dixerent route.

As is well known, AR processes with roots on the complex unit circle are non-stationary, and are actually more interesting than $A R$ processes with a 
real valued unit root, because these processes display a persistent cyclical behavior. Thus, if there exist persistent business cycles, it seems that the data generating process involved is more compatible with an $A R(M A)$ process with complex conjugate unit roots than with a real unit root and/or roots outside the complex unit circle.

In this paper we analyze the asymptotic properties of a standardized version of the periodogram for ARMA processes with complex unit roots in the AR lag polynomial, and derive a nonparametric test of the complex unit root hypothesis against the stationarity hypothesis. This test will be applied to US unemployment time series data ${ }^{1}$ in order to see whether this series has complex unit roots in the business cycle frequencies.

\section{AR (2) P rocesses with Complex U nit R oots}

\subsection{Introduction}

Consider the $A R(2)$ process

$$
y_{\mathrm{t}}=2 \cos (\dot{A}) y_{\mathrm{t}_{\mathrm{i}} 1} \text { i } \mathrm{y}_{\mathrm{t}_{\mathrm{i}} 2}+{ }^{1}+\mathrm{u}_{\mathrm{t}} \text {; }
$$

where $u_{t}$ is i.i.d. $\left(0 ;{ }^{3 / 2}\right)$ with $E j u_{t}{ }^{2+ \pm}<1$ for some $\pm>0 ;{ }^{1}$ is a constant, and $A$ $2\left(0 ; 1 / 4\right.$. Throughout this paper we assume that $y_{t}$ is observable for $\mathrm{t}=1 ; ;: ; ; \mathrm{n}$ : The $A R$ lag polynomial $\mathbb{O}(\mathrm{L})=1 ; 2 \cos (\hat{A}) \mathrm{L}+\mathrm{L}^{2}$ can be written as $\Theta(L)=(1 ; \exp (i A ́) L)(1 ; \exp (; i A ́) L)$, hence $\Theta(L)$ has two roots on the complex unit circle, $\exp (i \dot{A})=\cos (A))+i \sin (\dot{A})$; and its complex conjugate $\exp ($; i $\dot{A})=\cos (\hat{A})$; i $\sin (\hat{A}) ;$ provided that $\sin (\dot{A}) \Leftrightarrow 0$ : The latter condition will be assumed throughout the paper, because otherwise either $\cos (\hat{A})=1$; which implies that $y_{t}$ is I $(2)$, or $\cos (\hat{A})=i 1$; which implies that $y_{t}+y_{t_{i}}$ is I (1): .

Note that (1) generates a persistent cycle of 21/4Á periods. If Á $2\left(1 / 42^{1} / 4\right.$; the cycle length is less than two periods. Such short cycles are unlikely to occur in macroeconomic time series, and if they occur, they are dic cult, if not impossible, to distinguish from random variation. This is the reason for only considering the case Á $2\left(0 ;{ }^{1 / 4}\right.$ :

\footnotetext{
${ }^{1}$ The empirical application involved has been conducted with the author's free software package EasyReg (Version 1.28), which is downloadable from web page:

http:/ / econ.la.psu.edu/ hbierens/ EA SY RE G.HT M

The monthly unemployment time series involved is included in the E asyReg database.
} 
As is well known, the general solution of the dixerence equation (1) is a linear combination of a particular solution, which can be obtained by backwards substitution, and the solution of the homogenous equation $z_{t}=$ $2 \cos (\hat{A}) z_{t_{i}} 1$ i $z_{t_{i}}$ : As has been shown by Gregoir $(1999 a, b, c)$, using the operator

$$
S_{t}(\dot{A}) u_{t}=X_{j=1}^{t} \sin (A ́(t+1 ; j)) u_{j}
$$

for $t, 1$; the general solution is of the form:

LEM MA 1: Under data generating process (1), $y_{t}=S_{t}(A ́) u_{t}+d_{t} ;$ where and $d_{t}$ is a deterministic process of the form

$$
d_{t}=a \cos (A ́ t)+b \sin (\dot{A} t) ;
$$

with $a$ and $b$ real valued time invariant random variables depending on initial conditions.

It is a standard calculus exercise to show that

$$
\begin{aligned}
& S_{t}(\dot{A}) u_{t}=\sin (A ́(t+1))^{X^{t}} \cos (A ́ j) u_{j} i \cos (A ́(t+1))^{X^{t}} \sin (A ́ j) u_{j}
\end{aligned}
$$

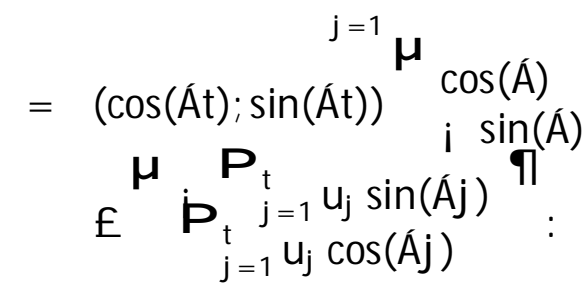

Moreover, it follows from the easy equalities

$$
\begin{aligned}
& X^{t} \sin (2 \dot{A} j)=\frac{\cos (\dot{A})}{2 \sin (\dot{A})}(1 ; \cos (2 \dot{A}(t+1))) i \frac{1}{2} \sin (2 \dot{A}(t+1)) \\
& X^{t} \cos (2 \dot{A} j)=\frac{\cos (\dot{A})}{2 \sin (\dot{A})} \sin (2 \dot{A}(t+1)) i \frac{1}{2}(1+\cos (2 \dot{A}(t+1))
\end{aligned}
$$

that for Á $2\left(0 ;{ }^{1} / 4\right.$;

$$
\frac{1}{n}_{j=1}^{X^{n}}(\cos (A ́ j))^{2}=\frac{1}{2}+\frac{1}{2 n}{ }_{j=1}^{X} \cos (2 \dot{A} j)=\frac{1}{2}+O(1=n)
$$




$$
\begin{aligned}
\frac{1}{n}_{j=1}^{X^{n}}(\sin (A ́ j))^{2} & =\frac{1}{2} i \frac{1}{2 n}_{j=1}^{X^{n}} \cos (2 A ́ j)=\frac{1}{2}+O(1=n) ; \\
\frac{1}{n}_{j=1}^{n} \sin (A ́ j) \cos (A ́ j) & =\frac{1}{2 n}{ }_{j=1}^{n} \sin (2 A ́ j)=O(1=n):
\end{aligned}
$$

Therefore, denoting ${ }^{2}$

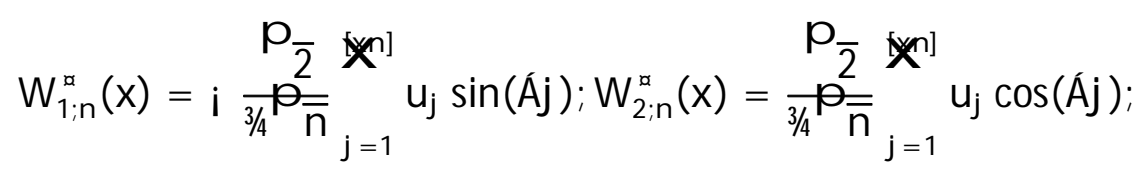

for $x 2$ [0;1]; it follows from Herrndorf's (1984) functional central limit theorem for ${ }^{\circledR}$-mixing processes that jointly ${ }^{3}$

$$
\left.\left.W_{1 ; n}^{a}\right) \quad W_{1} \text { and } W_{2 ; n}^{a}\right) \quad W_{2} ;
$$

where $W_{1}$ and $W_{2}$ are independent standard $W$ iener processes. See Billingsley (1968). The same applies to

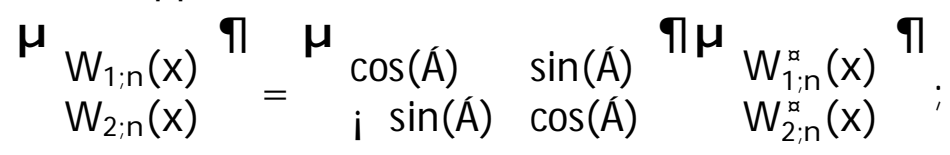

because the matrix involved is orthogonal. Consequently, we have

LEM MA 2: Under data-generating process (1),

$$
\begin{aligned}
y_{t}=\mathrm{p}_{\overline{\mathrm{n}}}= & \frac{{ }^{3 / 4} \mathrm{p}}{\sin (\hat{A})} \overline{\overline{2}}\left(\cos (\dot{A} \mathrm{t}) \mathrm{W}_{1 ; n}(\mathrm{t}=n)+\sin (\dot{A} \mathrm{t}) \mathrm{W}_{2 ; n}(\mathrm{t}=n)\right) \\
& +\mathrm{O}_{\mathrm{p}}(1=\overline{\mathrm{p}})
\end{aligned}
$$

where

$$
\mu_{\substack{W_{1 ; n} \\ W_{2 ; n}}}{ }^{\mu} W_{1} \text { १ }
$$

on $[0 ; 1] ;$ with $W_{1}$ and $W_{2}$ independent standard $W$ iener processes. Moreover, the $O_{p}(1=\bar{n})$ remainder term is uniform in $t=1 ;: ; ; n$ :

\footnotetext{
${ }^{2}$ Thoughout this paper we adopt the convention that for $t<1$ the sum ${ }_{\mathrm{t}} \mathrm{P}_{\mathrm{j}=1}$ is zero.
${ }^{3}$ Following Billingsley (1968), throughtout this paper the double arrow ) indicates weak convergence of random functions, or convergence in distribution in the case of random variables. The single arrow ! indicates convergence in probability, unless otherwise stated.
} 
Thus, $\mathrm{y}_{\mathrm{t}} \stackrel{\mathrm{p}}{=} \overline{\mathrm{n}}$ takes the form of a linear function of $\sin (\dot{A} \mathrm{t})$ and $\cos (\dot{A} \mathrm{t})$; with random coel cients $W_{1 ; n}(t=n)$ and $W_{2 ; n}(t=n)$; respectively, plus a vanishing remainder term. Consequently, the series $y_{t}$ will display a rather smooth cyclical pattern, with a cycle of $2^{1 / / f A}$ periods. A typical example is the arti..cial time series displayed in Figure 1 . This time series is generated by $y_{t}=1: 9960534 y_{t_{i} 1}$ i $y_{t_{i}}+u_{t}$; with $u_{t}$ i.i.d. $N(0 ; 1)$, for $t=1 ;: ; ; 500$ : This series has a cycle of 100 periods.

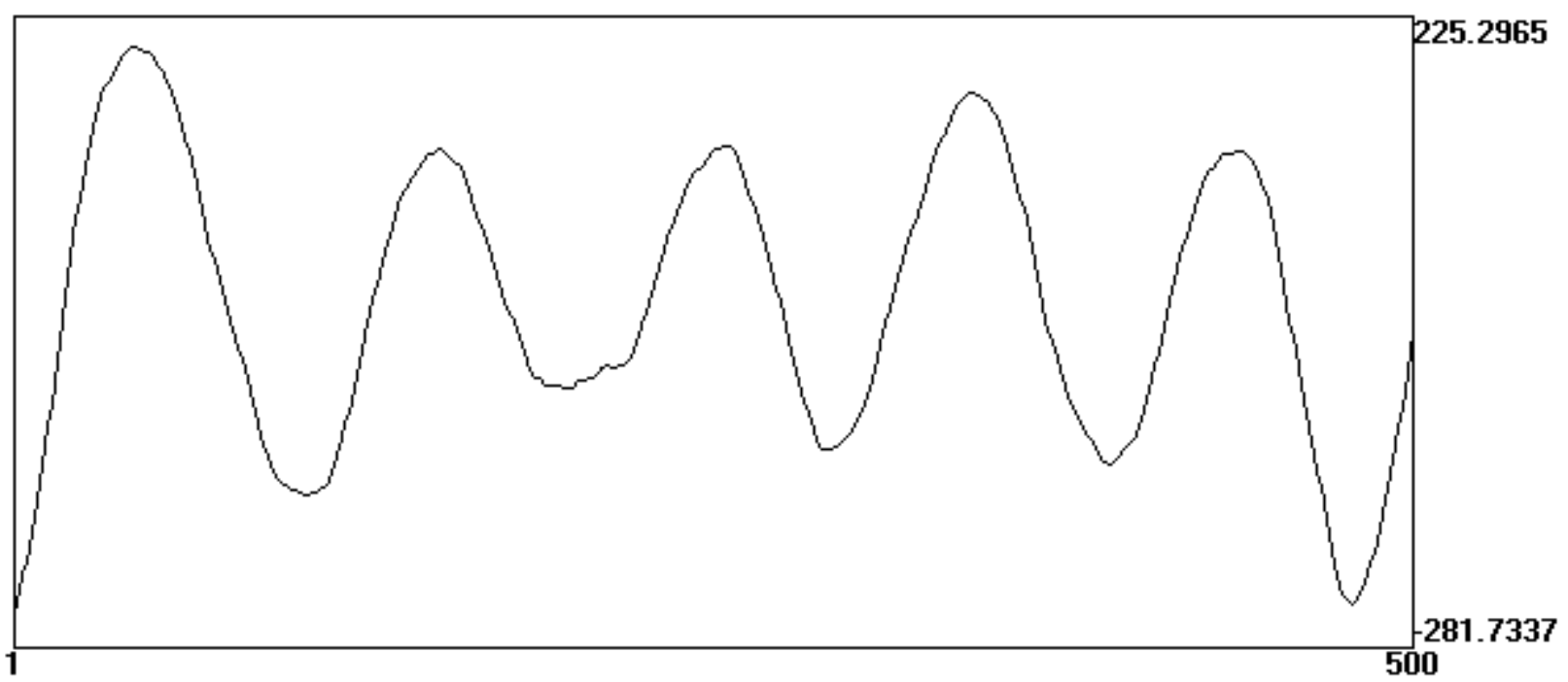

Figure 1: $A R(2)$ process with complex unit roots and a cycle of 100 periods

\subsection{Relaxing the i.i.d. error assumption}

The assumption that the errors $u_{t}$ in (1) are i.i.d. is not essential. We may replace it by:

ASSUMPTION 1: Let (1) hold, with $u_{t}$ a zero-mean stationary ARMA process: $u_{t}={ }^{\prime}(L) "{ }_{t}$; where " ${ }_{t}$ is i.i.d. $(0 ; 1) ; E \quad j "{ }_{t}{ }^{2+ \pm}<1$ for some $\pm>0$; ${ }^{\prime}(L)=P_{j=0}{ }^{\prime} L^{j}=\mu_{1}(L)=\mu_{2}(L)$ is a rational lag polynomial with all the 
roots of $\mu_{2}(L)$ outside the complex unit circle, and $\mu_{1}\left(\mathrm{e}^{\mathrm{i} A}\right) € 0 .{ }^{4}$

Then we can write

$$
\begin{aligned}
& u_{t}={ }^{\prime}\left(e^{i A \hat{A}}\right){ }^{\prime}{ }_{t}+{ }^{i} e^{i \hat{A}} i L^{\Phi^{\prime}(L) i{ }^{\prime}\left(e^{i \hat{A}}\right)} \frac{e^{i \hat{A}} i{ }_{t}}{}
\end{aligned}
$$

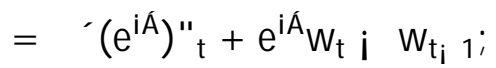

where

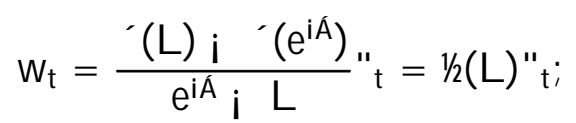

say. Since $1 / / L L)$ is a rational lag polynomial: $1 /(L L)=1 / 2(L)=\mu_{2}(L)$; where $1 / 2(L)$ is a ..nite-order lag polynomial, it follows that $w_{t}$ is a (complex-valued) stationary process.

Next, observe from (9) that

$$
\begin{aligned}
& { }_{j=1}^{X^{t}} \exp (i A ́ \dot{j}) u_{j}={ }^{\prime}\left(e^{i A ́}\right)_{j=1}^{X^{t}} \exp (i A ́ j){ }^{\prime}{ }_{j}+\exp (i A ́(t+1)) w_{t} \\
& \text { i } \exp (\mathrm{i} A ́) \mathrm{w}_{0} \text {; }
\end{aligned}
$$

and consequently

$$
\begin{aligned}
& X_{j=1}^{t} \cos (\hat{A} j) u_{j}=\operatorname{Re}^{i}{ }{ }^{\prime}\left(e^{i \hat{A})}{ }^{\phi X^{t}}{ }_{j=1} \cos (A ́ j){ }^{\prime \prime}{ }_{j}\right.
\end{aligned}
$$

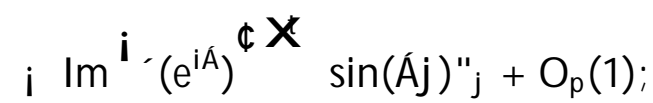

$$
\begin{aligned}
& { }_{j=1}^{X^{t}} \sin (A ́ j) u_{j}=R e^{i}{ }^{\prime}\left(e^{i A ́}\right)^{\phi X^{t}} \sin _{j=1}(A ́ j){ }^{\prime}{ }_{j} \\
& +\operatorname{Im}^{\mathrm{i},}\left(\mathrm{e}^{\mathrm{i} \hat{A})}{ }^{\phi X^{\mathrm{t}}} \cos (\hat{A} \mathrm{j})\right)_{j}+\mathrm{O}_{p}(1) ;
\end{aligned}
$$

where the $O_{p}(1)$ term is due to the stationarity of $w_{t}$ : Thus

\footnotetext{
${ }^{4}$ Since $\mu_{1}(\mathrm{~L})$ is real valued, all complex-valued roots come in conjugate pairs. Hence $\mu_{1}\left(\mathrm{e}^{\mathrm{iA}}\right) \Leftarrow 0$ implies $\mu_{1}\left(\mathrm{e}^{\left.\mathrm{i}{ }^{\mathrm{A}}\right)} \in 0\right.$; and vice versa.
} 
LEMMA 3: Let Assumption 1 hold. Rede..ne $3 / 4$ as

$$
3 / 4={ }^{-}\left(e^{i A ́}\right)^{-} ;
$$

and rede. ne $W_{1 ; n}$ and $W_{1 ; n}$ as

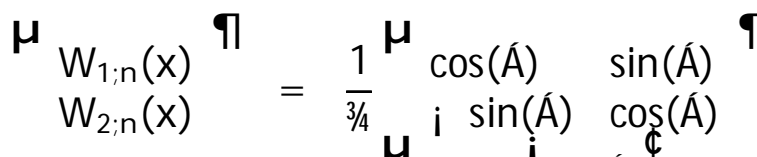

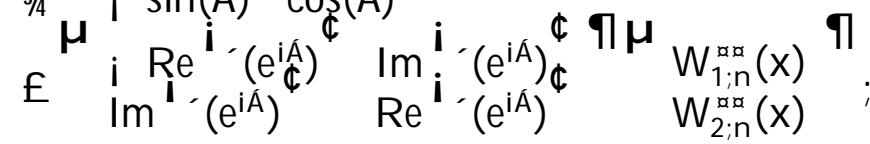

where

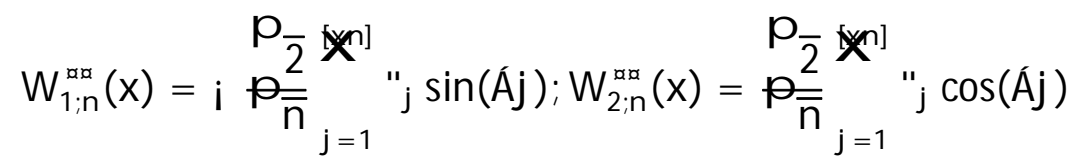

Then the result of Lemma 2 goes through. Moreover, (10) is related to the spectral density

$$
\pm(\gg)=\frac{1}{21 / 4}_{s=i 1}^{X} \cos (\gg S){ }_{j=0}^{X} \prime_{j}^{\prime} j+j s j
$$

of $u_{t}$, as follows: $\exists(A ́)=\frac{1}{2^{2} / 4} j^{\prime}(\exp (i \hat{A})) j^{2}=\frac{3 / 4}{2^{12} / 4}$

The proof of the latter is a standard exercise, and therefore left to the reader.

\subsection{Filtering}

The argument in the previous subsection also implies that, for example, differencing of $y_{t}$ does not eliminate the cycle, because the dixerence operator $1 ; \mathrm{i}$ changes ' $(\mathrm{L})$ to ${ }_{\mathrm{x}}(\mathrm{L})=(1 ; \mathrm{L}){ }^{\prime}(\mathrm{L})$; which still satis..es A ssumption 1. The same applies to any other linear ..Iter $i(L)$ with $i\left(e^{i \dot{A}}\right) \in 0$ : In order to show how (8) changes if $y_{t}$ is replaced by $i(L) y_{t}$, denote ${ }_{x}^{\prime}(L)=i(L)^{\prime}(L)$. Then

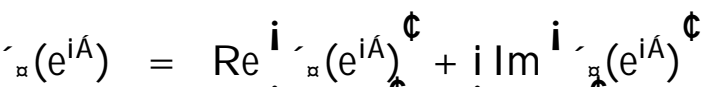

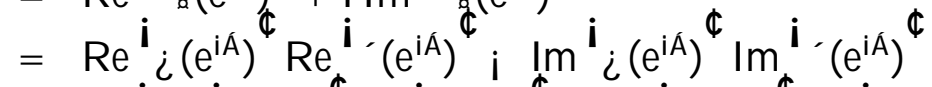

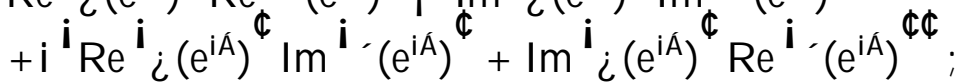


hence, denoting

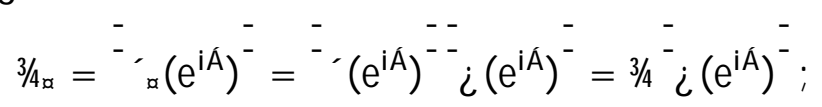

we have

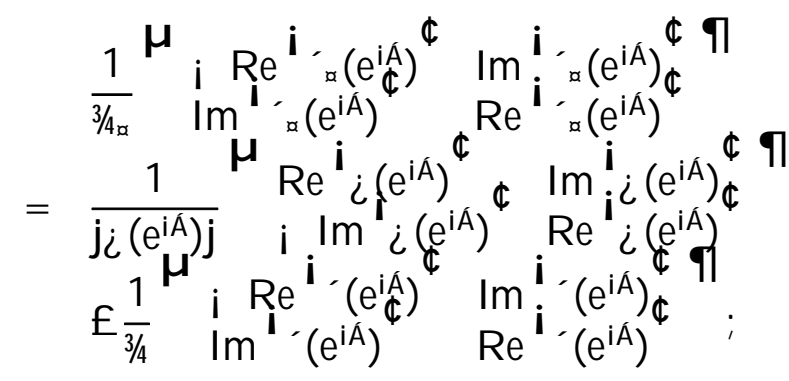

Therefore, it follows from Lemma 3, with ' $(L)$ replaced by $i(L)^{\prime}(L)$ :

LEMMA 4: Let $i(L)$ be a linear ..Iter satisfying $i\left(e^{i A ́}\right) \in 0$ : Under Assumption 1 ,

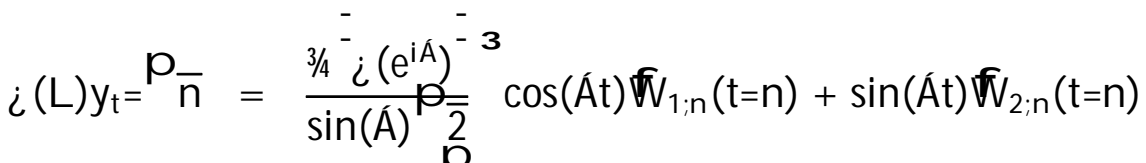

$$
\begin{aligned}
& +O_{p}(1=\bar{n}) \text {; }
\end{aligned}
$$

where $3 / 4={ }^{-},\left(e^{\mathrm{i} \dot{A})^{-}}\right.$, and

$$
\tilde{A}_{\substack{1 ; n \\ W_{2 ; n}}}, \mu_{W_{1}}{ }^{q} ;
$$

on $[0 ; 1]$; with $W_{1}$ and $W_{2}$ the same as before.

Strictly speaking, the result in Lemma 4 also applies to the double difference .. Iter $i(L)=(1 ; L)^{2}=1 ; 2 L+L^{2}=\phi^{2}$ : However, in practice this ...ter would wipe out a complex unit root in $\phi^{2} y_{t}$ if the complex unit root involved corresponds to a business cycle frequency. For example, the AR (2) lag polynomial of the process $y_{t}$ displayed in Figure 1 is $1 ; 1: 9960534 L+L^{2}$, which is numerically too close to $1 \mathrm{i} 2 \mathrm{~L}+\mathrm{L}^{2}$ to be distinguishable, hence the $A R$ and $M A$ lag polynomials of the resulting AR M A $(2 ; 2)$ process $\phi^{2} y_{t}$ will approximately cancel out, causing $\phi^{2} y_{t}$ to look like a white noise process. 


\section{Frequency A nalysis}

The periodogram $1 / \not \rightarrow(»)$; say, of a time series $y_{t}$ is de. ned by

$$
1 / \beta(\gg)=\frac{2}{n} 4^{2}{ }_{t=1}^{X^{n}} y_{t} \cos (\gg t)+{ }_{2}{ }^{\tilde{A}} X_{t=1}^{n} y_{t} \sin (\gg t) \quad 5:
$$

for » $2(0 ; 1 / 4)$ and odd $n$ : See Fuller (1976, Chapter 7).

If $y_{t}$ is a stationary linear process, say:

$\mathrm{y}_{\mathrm{t}}={ }^{1}+{ }^{\prime}(\mathrm{L}){ }^{\mathrm{t}} \mathrm{t}$; where ${ }^{\prime}(\mathrm{L})$ and ${ }_{\mathrm{t}} \mathrm{t}$ are the same as in Assumption $1,(14)$

then for ..xed Á $2(0 ; 1 / 4$;

$$
1 / \not(A ́)) \quad 21 / 4+(A ́) \hat{A}_{2}^{2}
$$

where $\pm(A)$ is the spectral density of $y_{t}$. See (13) and Fuller (1976, Theorem 7.1.2, p. 280). As is not hard to verify, this result is due to the fact that un-

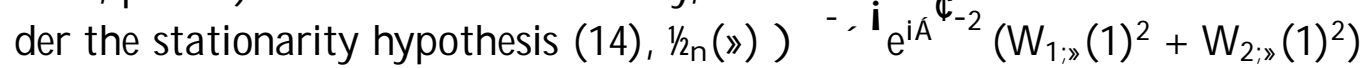
pointwise in » $2(0 ; 1 / 4)$; where $W_{1 ; \gg}$ and $W_{2 ;>}$ are independent standard $W$ iener processes depending on $»$, which are also independent across the $\gg ' s$; and

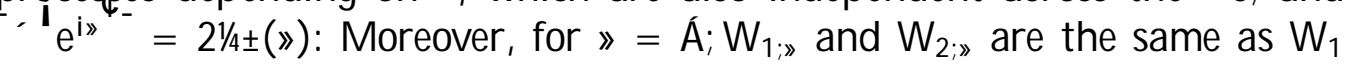
and $W_{2}$ in the complex unit root case. Furthermore, since

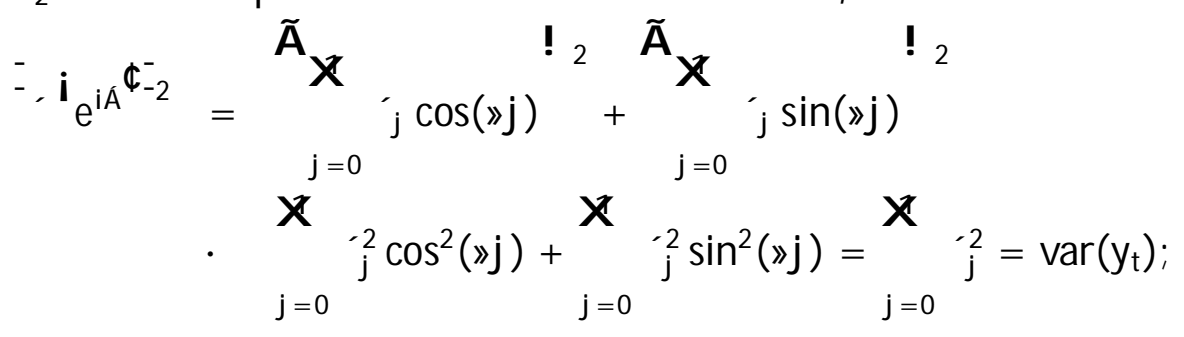

it follows that under the stationarity hypothesis,

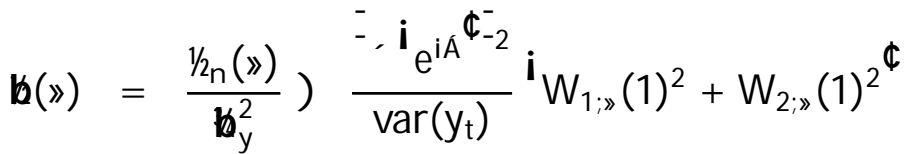

$$
\begin{aligned}
& \text { - } \quad W_{1 ; \gg}(1)^{2}+W_{2 ; \gg}(1)^{2} \text {; }
\end{aligned}
$$

where

$$
b_{y}^{2}=\frac{1}{n_{i} 1}{ }_{t=1}^{n}\left(y_{t} i \quad y\right)^{2}
$$


is the sample variance, with 8 the sample mean.

The main idea in this paper is to use the standardized periodogram b(z)) as the basis for a nonparametric test of the complex unit root hypothesis against the stationarity hypothesis, because in the complex unit root case the properties of $b(\$)$ are quite dixerent from the stationary case. This is illustrated in Figures 2 and 3 . Figure 2 displays the periodogram of the complex unit root process plotted in Figure 1 . Figure 3 displays the periodogram of the stationary Gaussian AR(2) process $y_{t}=1: 411423 y_{t_{i}} 1$ i :5y $y_{t_{i}}+u_{t}$; $t=1 ;: ; 500$; where the $u_{t}$ 's are i.i.d. $N(0 ; 1)$. The lag polynomial of this $A R(2)$ process has complex roots outside the unit circle, corresponding to a (vanishing) cycle of 100 periods.

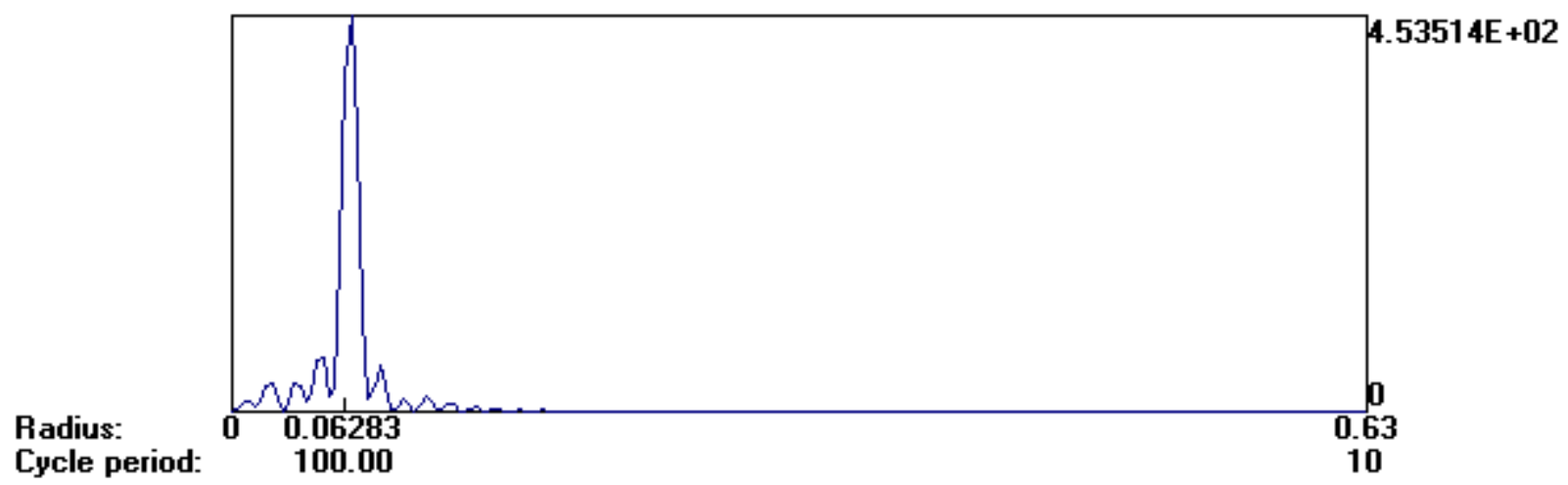

Figure 2: Periodogram of the complex unit root process plotted in Figure 1

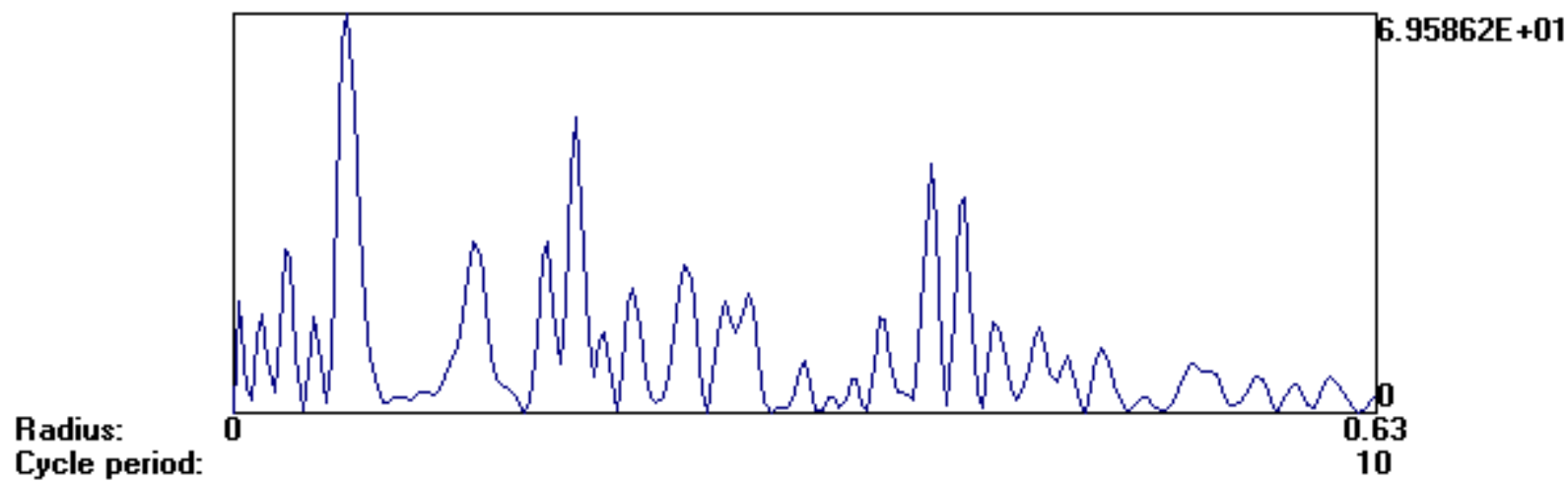


Figure 3: Periodogram of a stationary AR(2) process with complex roots and a cycle of 100 periods

We see that the two periodograms are very distinct, in shape as well as in scale. In particular, the periodogram of the stationary process has many more, and more widely spread, peaks than the periodogram of the complex unit root process, and the peaks are much lower than in the latter case.

The following lemma, which is proved in the appendix, explains the differences between these two cases.

LEM MA 5: Under Assumption 1,

$$
\mathrm{y}=\frac{1}{\mathrm{n}}_{\mathrm{t}=1}^{\mathrm{X}} \mathrm{y}_{\mathrm{t}}=\mathrm{O}_{\mathrm{p}}(1) \text {; }
$$

and

$$
\left.{\frac{1}{n^{2}}}_{t=1}^{x} y_{t}^{2}\right){\frac{3 / 4}{4 \sin ^{2}(A)}}_{0}^{\mu Z_{1}} W_{1}(x)^{2} d x+{ }_{0}^{Z_{1}} W_{2}(x)^{2} d x \text { : }
$$

Moreover,

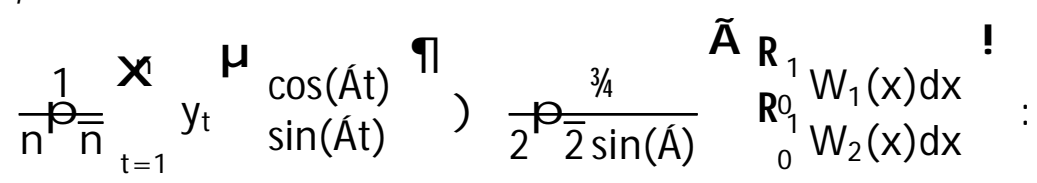

Furthermore, for ..xed $\gg 2(0 ; A ́)\left[\left(\hat{A}^{\prime}{ }^{1 / 4}\right.\right.$;

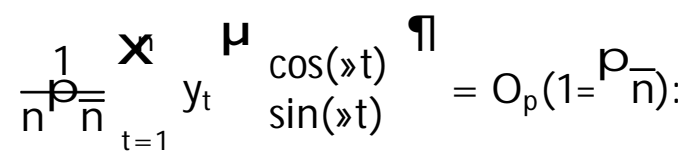

Lemma 5 implies that in the complex unit root case b(y) $)=n$ has a sharp spike at $\gg=A$; with height asymptotically distributed as

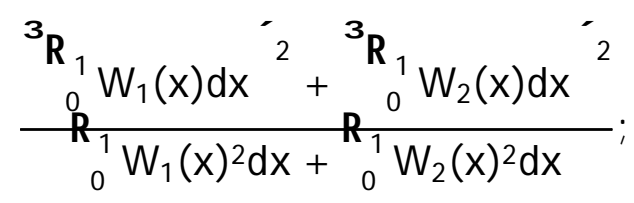

and asymptotically zero elsewhere, whereas (15) implies that under the stationarity hypothesis, b(z)) is bounded away from zero, and asymptotically 
bounded from above by independent $\hat{A}_{2}^{2}$ random variables, pointwise in $\gg 2$ $(0 ; 1 / 4)$ :

Summarizing, we have shown:

THEOREM 1: Consider the standardized periodogram

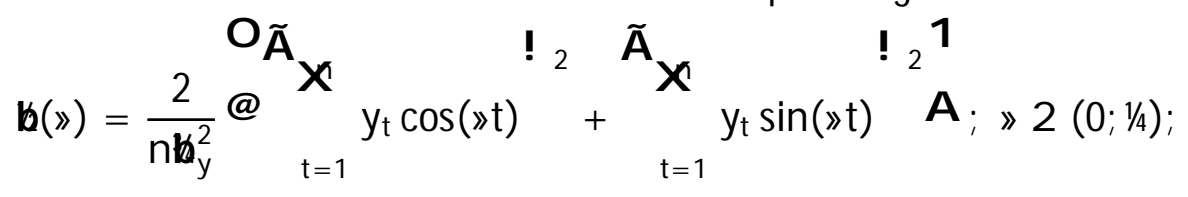

where ${ }_{y}^{2}$ is the sample variance. Under Assumption 1 ,

$$
\begin{aligned}
& \left.\frac{B(2))}{n}\right) \frac{{ }_{0}^{R_{1}} W_{1}(x) d x^{2}+{ }_{{ }_{1}}{ }_{R_{1}} W_{2}(x) d x^{\prime}{ }^{2}(x)^{2} d x+{ }_{0}{ }_{0} W_{2}(x)^{2} d x}{{ }_{0} W_{1}} \text { if } »=\dot{A} \text {; } \\
& \frac{b(p))}{\mathrm{n}}=\mathrm{O}_{\mathrm{p}}{ }^{\mathrm{i}}{ }_{1}{ }^{\mathrm{p}} \overline{\mathrm{n}}^{\Phi} \text { if } \gg \theta \dot{A} \text {; }
\end{aligned}
$$

pointwise in $\gg 2(0 ; 1 / 4)$; where $W_{1}$ and $W_{2}$ are independent standard $W$ iener processes.

Under the stationarity hypothesis (14),

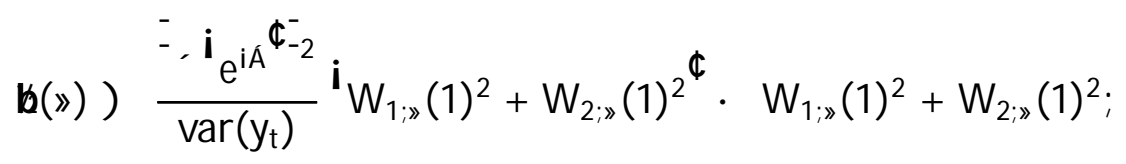

pointwise in $\gg 2\left(0 ; 1 / 4\right.$; where $f\left(W_{1 ; \gg ;} W_{2 ; \gg}\right) ; \gg 2(0 ; 1 / 4 g$ is a collection of independent bivariate standard $W$ iener processes. Moreover, for $\gg=\dot{A}$; $\mathrm{W}_{1 ; \gg}=\mathrm{W}_{1}$ and $\mathrm{W}_{2 ; \gg}=\mathrm{W}_{2}$ :

\section{M ultiple Cycles}

\subsection{The state space case}

The periodograms of macroeconomic time series often display multiple peaks in the business cycle frequencies. If $k$ of these peaks are due to complex unit roots, then one way of modelling the process involved is as an AR (2k) process with all the roots of the AR lag polynomial on the complex unit circle. However, as is already clear from Figure 1, the plots of such processes are 
very smooth; much smoother than for most economic time series. Therefore, in ..rst instance we propose to model these time series as a state space model of aggregates of ARMA processes with dixerent single pairs of complexconjugate unit roots, plus a stationary AR M A process representing the noise. The AR (2k) case will be considered in the next subsection.

ASSUMPTION 2: The data-generating process is: $y_{t}=P_{j=0} y_{j ; t}$; where $\mathrm{y}_{0 ; t}={ }^{1} 0+{ }^{\prime}{ }_{0}(\mathrm{~L}){ }_{0 ; \mathrm{t}}$ satis..es the conditions in (14), and for $\mathrm{j}=1 ; \ldots ; \mathrm{k}$; $y_{j ; t}=2 \cos \left(A_{j}\right) y_{j ; t_{i} 1} i y_{j ; t_{i}}+{ }^{1}{ }_{j}+{ }^{\prime}(L){ }_{j}{ }_{j ; t}$; with $0<A_{1}<:::<A_{k}<1 / 4$ The lag polynomials ' ${ }_{j}(L)$ are rational: ' $\left.{ }_{j}(L)={ }_{1 ; j} L\right)={ }_{2 ; j}(L)$; with ' ${ }_{2 ; j}(L)$ having all its soots outside the unit circle, and the $(" 1 ; t ; \ldots ; ; " k ; t)$ 's are i.i.d. $(0 ; 1)$, with $E \mathrm{j}^{\mathrm{j}} \mathrm{j} ; \mathrm{t}^{2+ \pm}<1$ for some $\pm>0$ :

A dmittedly, the assumption that the " $j ;$ it $s$ are uncorrelated across the $j$ 's is quite restrictive, but is needed in order to derive nuisance-free asymptotic null distributions of the tests we are going to propose.

The process $\mathrm{y}_{0 ; \mathrm{t}}$ will only play a role under the alternative hypothesis of stationarity, which corresponds to the case $k=0$ :

It follows straightforwardly from Lemma 3 that under Assumption 2,

$$
\begin{aligned}
y_{t}=p_{\bar{n}}= & { }^{X^{k}} \frac{p^{3 / 4}}{\overline{2} \sin \left(\hat{A}_{j}\right)}\left(\cos \left(\dot{A}_{j} t\right) W_{1 ; j ; n}(t=n)+\sin \left(\dot{A}_{j} t\right) W_{2 ; j ; n}(t=n)\right) \\
& +O_{p}(1=\bar{p} \bar{n}) ;
\end{aligned}
$$

hence (17) still holds, and (18) becomes

$$
\left.{\frac{1}{n^{2}}}_{t=1}^{X^{n}} y_{t}^{2}\right) \frac{1}{4}_{j=1}^{X^{k}}{\frac{3 / 4}{\sin ^{2}\left(\dot{A}_{j}\right)}}_{0}^{\mu Z_{1}} W_{1 ; m}(x)^{2} d x+{ }_{0}^{Z_{1}} W_{2 ; m}(x)^{2} d x ;
$$

where

$$
3 / 4=j^{\prime} j\left(\exp \left(i A_{j}\right)\right) j
$$

and

$$
\left.\left(W_{1 ; j ; n} ; W_{2 ; j ; n}\right)^{0}\right) \quad W_{j}=\left(W_{1 ; j} ; W_{2 ; j}\right)^{0}
$$

jointly, with $W_{1} ;:: ; W_{k}$ independent bivariate standard $W$ iener processes. Note that without the assumption that the " $j ; t$ 's are uncorrelated across the $\mathrm{j}$ 's, the $\mathrm{W}_{\mathrm{j}}$ 's would be dependent, but that is the only dixerence. 
Except for parts (22) and (23), the following results follow straightforwardly from Lemma 5:

THEOREM 2: Let $!_{j}=\left(1={ }^{p} \overline{2}\right)^{3 / 4}=\sin \left(\dot{A}_{j}\right)$ : Under Assumption 2 ,

$$
\begin{aligned}
& y_{t}=p_{\bar{n}}={ }^{k} !_{m}\left(\cos \left(\dot{A}_{m} t\right) W_{1 ; m ; n}(t=n)+\sin \left(\dot{A}_{m} t\right) W_{2 ; m ; n}(t=n)\right) \\
& +O_{p} i_{1}={ }_{n} \phi \text { : }
\end{aligned}
$$

and consequently,

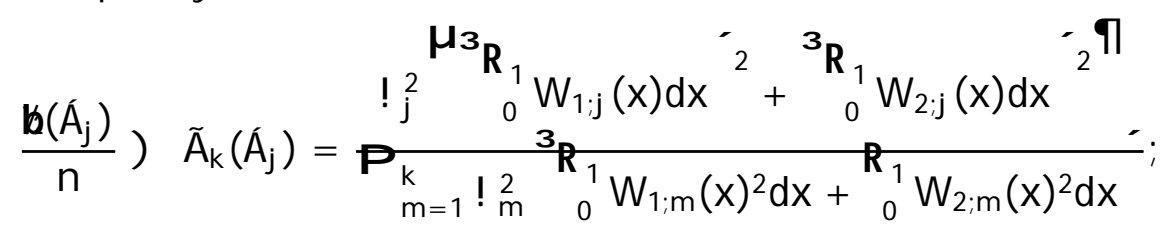

jointly for $j=1 ; \ldots ; k$ : Hence, $\left.\left.\max _{j=1 ;: ; k} \quad A_{j} \hat{A}_{j}\right)=n\right) \quad \max _{j=1 ; \ldots ; k} \tilde{A}_{k}\left(\hat{A}_{j}\right)$ and

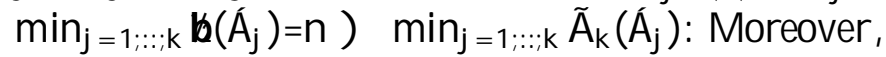

$$
\max _{j=1 ;: ;: k} \tilde{A}_{k}\left(\dot{A}_{j}\right), B_{k} ; \min _{j=1 ;: ;: ; k} \tilde{A}_{k}\left(\dot{A}_{j}\right) \cdot B_{k} ;
$$

where

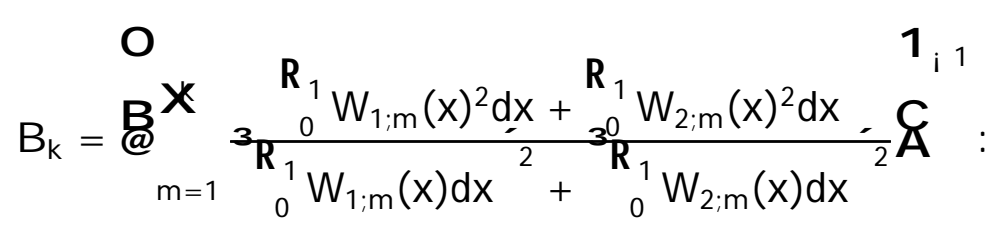

Furthermore,

pointwise in » $2\left(0 ;{ }^{1} / 4 \mathrm{nf}^{\prime} \hat{A}_{1} ; \ldots ; \hat{A}_{k} g\right.$ :

$$
\frac{b(2))}{n}=O_{p}(1=\stackrel{p}{n}) ;
$$

Theorem 2 suggests to test the complex unit root hypothesis:

$\mathrm{H}_{0}$ : A ssumption 2 holds for given $\mathrm{k}$ and $\hat{A}_{1}=\hat{A}_{0 ; 1} ; \ldots ; \dot{A}_{k}=\hat{A}_{0 ; k} ;$ by using the test statistic

$$
B_{k}=\max _{j=1 ;: ; ; k} B\left(\hat{A}_{0 ; j}\right)=n
$$


with ${ }^{\circledR} f 100 \%$ critical values $\underline{c}_{k}(\circledR)$; say, based on the lowerbound $B_{k}$ of the asymptotic null distribution of $\dot{B}_{k}$ :

$$
\mathrm{P}\left(\mathrm{B}_{\mathrm{k}} \cdot \underline{\mathrm{C}}_{\mathrm{k}}(\AA)\right)={ }^{\circledR} \text { : }
$$

In Table 1 we present the critical values $\underline{c}_{k}(\circledR)$ for $k=1 ;: ; ; 10$, and $\AA=$ $0: 05,0: 10$, which have been computed by Monte Carlo simulation. ${ }^{5}$

\begin{tabular}{|c|c|c|}
\hline Table 1: Values of $\underline{c}_{k}($ & $\AA=0: 05$ & $B=0: 10$ \\
\hline & $0: 1403$ & $0: 2411$ \\
\hline & 0:0667 & $0: 1146$ \\
\hline & 0:0441 & 0:0732 \\
\hline & 0:0313 & 0:0519 \\
\hline & 0:0249 & 0:0409 \\
\hline & 0:0210 & $0: 0337$ \\
\hline & 0:0177 & $0: 0287$ \\
\hline & 0:0154 & $0: 0250$ \\
\hline & 0:0137 & $0: 022$ \\
\hline & 0:012 & \\
\hline
\end{tabular}

Given that $k$ and $A_{0 ; 1} ;:: ; \hat{A}_{0 ; k}$ are speci..ed in advance, this test is consistent against the stationarity hypothesis, as well as the hypothesis that none of the given values of $A_{0 ; 1} ; \ldots ; \hat{A}_{0 ; k}$ correspond to the ones in Assumption 2 . In particular, for the speci..ed frequencies $\hat{A}_{0 ; 1} ;:: ; \hat{A}_{0 ; k}$ under the null hypothesis, we have under stationarity that for $M>0$, and independent $\hat{A}_{2}^{2}$ variates $\hat{A}_{2}^{2}(1) ; \ldots .: ; \hat{A}_{2}^{2}(k)$;

$$
\begin{aligned}
& P^{3} n B_{k}, M^{\prime} ! P \max _{\mu=1 ;: ; k}^{\mu} \frac{j^{\prime}\left(\exp \left(i \dot{A}_{0 ; j}\right)\right) j_{j}}{\operatorname{var}\left(y_{t}\right)} \hat{A}_{2}^{2}(j), M^{\text {q }} \\
& \text { - } P \max _{j=1 ; ; ; ; k} \hat{A}_{2}^{2}(j), M \\
& =1_{i}\left(1_{i} \exp (i \mathrm{M}=2)\right)^{k} \text {; }
\end{aligned}
$$

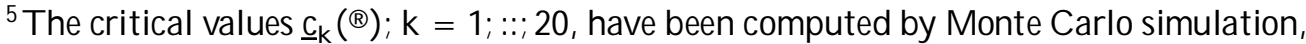
on the basis of $10 ; 100$ replications of 20 independent Gaussian randomp walks $75 ; t=$

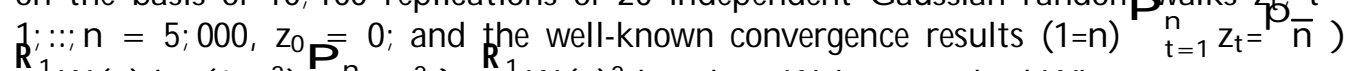
$\left.{ }_{0}^{R_{1}} W(x) d x ;\left(1=n^{2}\right)_{t=1}^{P} z_{t}^{2}\right) \quad{ }_{0}^{R_{1}} W(x)^{2} d x$; where $W$ is a standard $W$ iener process.
} 
where the last step follows from the fact that the $\hat{A}_{2}^{2}$ distribution is the same as the exponential distribution with expected value 2 . Setting

$$
\circledR=1 ; \quad\left(1 ; \exp \left(i \bar{c}_{k}(\circledR)=2\right)\right)^{k}, \quad \bar{c}_{k}(\circledR)=i 2 \ln ^{3} 1 ; \quad(1 ; @)^{1=k}
$$

then yields an upperbound $\tau_{\mathrm{k}}\left({ }^{\circledR}\right)$ of the asymptotic $\circledast f 100 \%$ critical value of a test of the stationarity hypothesis, with test statistic $n B_{k}$ :

\subsection{The AR(2k) case}

Consider the $\mathrm{AR}(2 \mathrm{k})$ model with $\mathrm{k}$ pairs of complex conjugate unit roots:

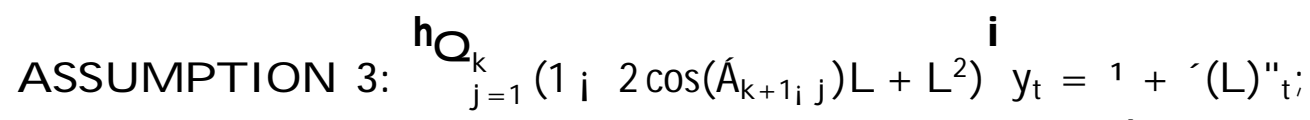
where ' $(L)$ and " $t$ are the same as in Assumption 1 , and $0<A_{1}<:::<$ $\hat{A}_{k}<1 / 4$

Let $u_{t}={ }^{\prime}(L) " t$ : It follows similarly to Lemma 1 that

$$
y_{t}=S_{t}\left(\dot{A}_{k}\right) S_{t}\left(\dot{A}_{k_{i} 1}\right)::::: S_{t}\left(\dot{A}_{1}\right) u_{t}+d_{t} ;
$$

where $S_{t}(A ́)$ is de..ned by (2), for each pair $\hat{A}_{1} ; \dot{A}_{2}$,

$$
S_{t}\left(\dot{A}_{2}\right) S_{t}\left(\dot{A}_{1}\right)={ }_{j=1}^{X^{t}} \sin \left(\dot{A}_{2}(t+1 ; j)\right) S_{j}\left(\dot{A}_{1}\right) ;
$$

and $d_{t}$ is a deterministic process of the type (3).

Next, let

$$
C_{t}(\hat{A}) u_{t}=X_{j=1}^{X^{t}} \cos \left(\dot{A}_{1}(t+1 ; j)\right) u_{t} ;
$$

and let for each pair $\hat{A}_{1} ; \dot{A}_{2}$;

$$
S_{t}\left(\dot{A}_{2}\right) C_{t}\left(\dot{A}_{1}\right)=\prod_{j=1}^{X t} \sin \left(\dot{A}_{2}(t+1 ; j)\right) C_{j}\left(\dot{A}_{1}\right):
$$

Then we have: 
LEMMA 6:

$$
\begin{aligned}
& S_{\mathrm{t}}\left(\hat{A}_{2}\right) S_{\mathrm{t}}\left(\hat{A}_{1}\right)=\left({ }_{1}^{\circ}\left(\hat{A}_{2} ; \dot{A}_{1}\right) ; \pm_{1}\left(\hat{A}_{2} ; \hat{A}_{1}\right)\right)\left(C_{\mathrm{t}}\left(\hat{A}_{2}\right) ; C_{\mathrm{t}}\left(\hat{A}_{1}\right)\right) \\
& \text { i }{ }^{\circ}\left(\hat{A}_{2} ; \dot{A}_{1}\right)\left(S_{t}\left(\dot{A}_{2}\right) \text {; } S_{t}\left(\dot{A}_{1}\right)\right) \\
& + \pm_{2}\left(\hat{A}_{2} ; \dot{A}_{1}\right)\left(S_{t}\left(\dot{A}_{2}\right)+S_{t}\left(\hat{A}_{1}\right)\right) \text {; } \\
& \mathrm{S}_{\mathrm{t}}\left(\hat{A}_{2}\right) \mathrm{C}_{\mathrm{t}}\left(\hat{A}_{1}\right)=\mathrm{i}\left({ }^{\circ}{ }_{2}\left(\hat{A}_{2} ; \dot{A}_{1}\right)+ \pm_{2}\left(\hat{A}_{2} ; \dot{A}_{1}\right)\right)\left(\mathrm{C}_{\mathrm{t}}\left(\hat{A}_{2}\right) ; \mathrm{C}_{\mathrm{t}}\left(\hat{A}_{1}\right)\right) \\
& \text { i }{ }^{\circ}\left(\hat{A}_{2} ; \dot{A}_{1}\right)\left(S_{t}\left(\dot{A}_{2}\right) \text {; } S_{t}\left(\dot{A}_{1}\right)\right) \\
& \text { i } \pm_{1}\left(\hat{A}_{2} ; \dot{A}_{1}\right)\left(S_{\mathrm{t}}\left(\hat{A}_{2}\right)+S_{\mathrm{t}}\left(\dot{A}_{1}\right)\right) \text {; }
\end{aligned}
$$

where

$$
\begin{aligned}
& { }_{1}^{\circ}\left(\dot{A}_{2} ; \dot{A}_{1}\right)=\frac{1}{2} \frac{\cos \left(\dot{A}_{2}\right) \text { i } \cos \left(\hat{A}_{1}\right)}{\left(\cos \left(\hat{A}_{2}\right) ; \cos \left(\dot{A}_{1}\right)\right)^{2}+\left(\sin \left(\dot{A}_{2}\right) ; \sin \left(\hat{A}_{1}\right)\right)^{2}} ; \\
& { }_{2} 2\left(\dot{A}_{2} ; \dot{A}_{1}\right)=\frac{1}{2} \frac{\sin \left(\dot{A}_{2}\right) ; \sin \left(\dot{A}_{1}\right)}{\left(\cos \left(\dot{A}_{2}\right) ; \cos \left(\dot{A}_{1}\right)\right)^{2}+\left(\sin \left(\dot{A}_{2}\right) ; \sin \left(\dot{A}_{1}\right)\right)^{2}} ; \\
& \pm_{ \pm}\left(\hat{A}_{2} ; \dot{A}_{1}\right)=\frac{1}{2} \frac{\cos \left(\dot{A}_{2}\right) ; \cos \left(\dot{A}_{1}\right)}{\left(\cos \left(\hat{A}_{2}\right) ; \cos \left(\dot{A}_{1}\right)\right)^{2}+\left(\sin \left(\dot{A}_{2}\right)+\sin \left(\dot{A}_{1}\right)\right)^{2}} \\
& \pm_{2}\left(\dot{A}_{2} ; \dot{A}_{1}\right)=\frac{1}{2} \frac{\sin \left(\dot{A}_{2}\right)+\sin \left(\dot{A}_{1}\right)}{\left(\cos \left(\dot{A}_{2}\right) ; \cos \left(\dot{A}_{1}\right)\right)^{2}+\left(\sin \left(\dot{A}_{2}\right)+\sin \left(\dot{A}_{1}\right)\right)^{2}}:
\end{aligned}
$$

The proof of Lemma 6 is pretty tedious, but involves only elementary trigonometric operations, and is therefore omitted.

Lemma 6 implies that $y_{t}$ can we written as

$$
y_{t}={ }_{j=1}^{X^{k}}{ }_{j} S_{t}\left(\dot{A}_{j}\right) u_{t}+{ }_{j=1}^{X^{k}} \ddagger C_{t}\left(\dot{A}_{j}\right) u_{t}+d_{t} ;
$$

where the ${ }^{\circ}{ }_{j}$ 's and $f_{j}$ 's are constants depending on the $\hat{A}_{j}{ }^{\prime}$ 's. M oreover, similar to Lemma 2 it follows that there exist orthogonal $2 £ 2$ matrices $Q_{1} ; \ldots ; Q_{k}$ and constants $\cdot$ j such that

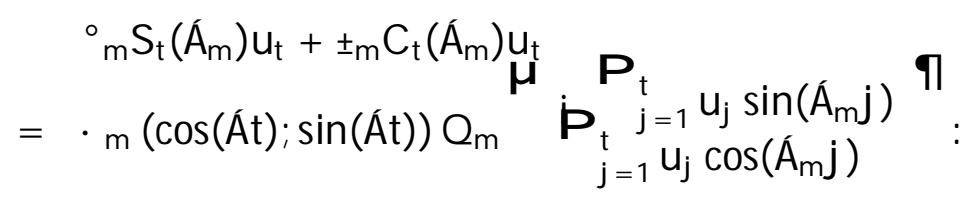


Furthermore, it follows from Lemma 3 that there exist orthogonal $2 f 2$ matrices $\mathrm{R}_{1} ; \ldots: ; \mathrm{R}_{\mathrm{k}}$ such that

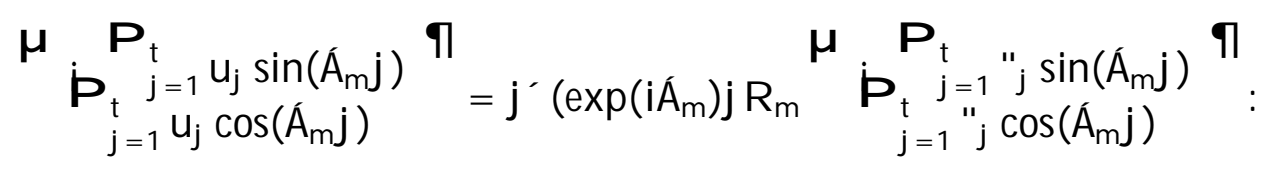

Therefore, de..ning

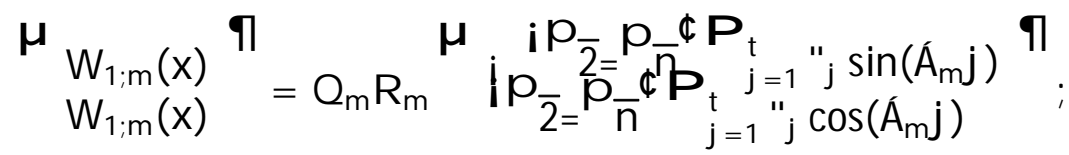

it follows that there exist constants $!_{\mathrm{j}}$ such that (21) carries over. Consequently,

THEOREM 3: A part from the de..nition of the constants ! $\mathrm{j}$; Theorem 2 holds under Assumption 3 as well.

This result also holds if we combine A ssumptions 2 and 3, i.e.

ASSUMPTION 4: Let $y_{t}=P_{K} h_{d_{k}=0} y_{j ; t}$; where $y_{0 ; t}$ is the same as in Assumption 2, and for $\mathrm{j}=1 ;: ; ; \mathrm{K}, \mathrm{Q}_{\mathrm{j}=1}\left(1 ; 2 \cos \left(\hat{A}_{\mathrm{k}+1_{\mathrm{i}} \mathrm{j}}\right) \mathrm{L}+\mathrm{L}^{2}\right) \mathrm{y}_{\mathrm{j} ; \mathrm{t}}=$ ${ }^{1}{ }_{j}+{ }^{\prime}(L) "{ }_{j ; t}$; where ${ }_{j}(L)$ and ${ }^{j} j$ t are the same as in Assumption 2 , and $0<A_{1}<:::<A_{k}<1 / 4$

Thus, in this case the processes $y_{j ; t} ; j=1 ;:: ; K$; have common complexconjugate unit roots. The condition in Assumption 2 that the " $j ; t^{\prime}$ 's are uncorrelated across the $\mathrm{j}$ 's is now no longer needed, because if the variance matrix of $\left(" 1 ; ; ;: ; ;{ }^{\prime} K_{; t}\right)^{0}$ is $\S$; say, we may without loss of generality replace

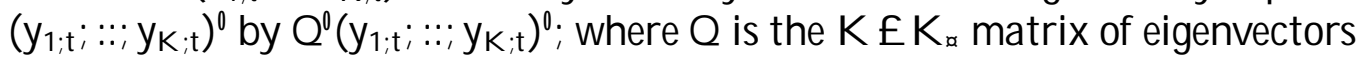
of $\S$ corresponding to the $K_{x}$ positive eigenvalues. Thus, without loss of generality we may assume that $\S=1$ :

Under Assumption 4 there exist constants ! j;m such that (21) becomes

$$
\begin{aligned}
y_{t}=p_{\bar{n}}= & x^{K} X^{k} !_{j ; m}\left(\cos \left(\dot{A}_{m} t\right) W_{1 ; j ; m ; n}(t=n)+\sin \left(\dot{A}_{m} t\right) W_{2 ; j ; m ; n}(t=n)\right) \\
& +O_{p}{ }_{i}{ }_{1}=p_{n}{ }^{\phi}:
\end{aligned}
$$




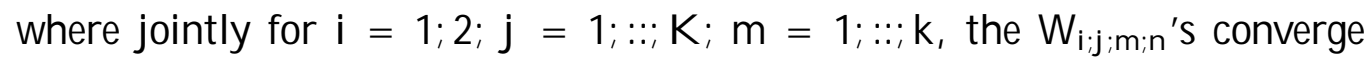
weakly to independent standard $\mathrm{W}$ iener processes $\mathrm{W}_{\mathrm{i} ; j ; \mathrm{m}}$ : Denoting

$$
W_{i ; m ; n}(x)=\frac{P_{j=1}^{K} !_{j ; m} W_{i ; j ; m ; n}(x)}{! m} ;
$$

where

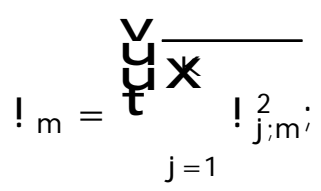

we now have that

THEOREM 4: Theorem 3 carries over under Assumption 4.

\section{A re Business Cycles Due to Complex Unit R oots?}

In conducting the test for complex unit roots, it is tempting to formulate the null hypothesis (24) by looking at the periodogram of the time series involved, and selecting the frequencies $\hat{A}_{0 ; 1} ;: \cdots ; \hat{A}_{0 ; k}$ corresponding to the $k$ highest peaks. However, this is akin to pretesting, and will axect the actual size and power of the test. The correct way of conducting the test is to formulate the null hypothesis prior to looking at the data. But all information about business cycles is based on empirical investigations [see for example Diebold and Rudebush 1999 and the references therein], so that even if we would choose $A_{0 ; 1} ; \ldots ; ; \hat{A}_{0 ; k}$ corresponding to the NBER business cycle dates and durations listed in Diebold and Rudebush (1999, Table 2.1, p.39), prior to looking at the periodogram, we would indirectly commit a pretesting-type of sin as well. In testing for seasonal unit roots this problem does not occur, of course, but is virtually impossible to avoid when testing for complex unit roots in the business cycle frequencies. In our empirical application we will therefore ignore this problem, and look at the periodogram ..rst, in order to determine potential complex unit root frequencies.

The time series we analyze is the monthly number of civilian unemployed for 15 weeks or more in the US, times 1000, from 1948.01 to 1999.07. This series is seasonally adjusted, but in order to be sure that there are no seasonal 
unit roots left, and to eliminate a possible unit root 1 as well, we have transformed the series to annual changes. The plot of the transformed series is displayed in Figure 4.

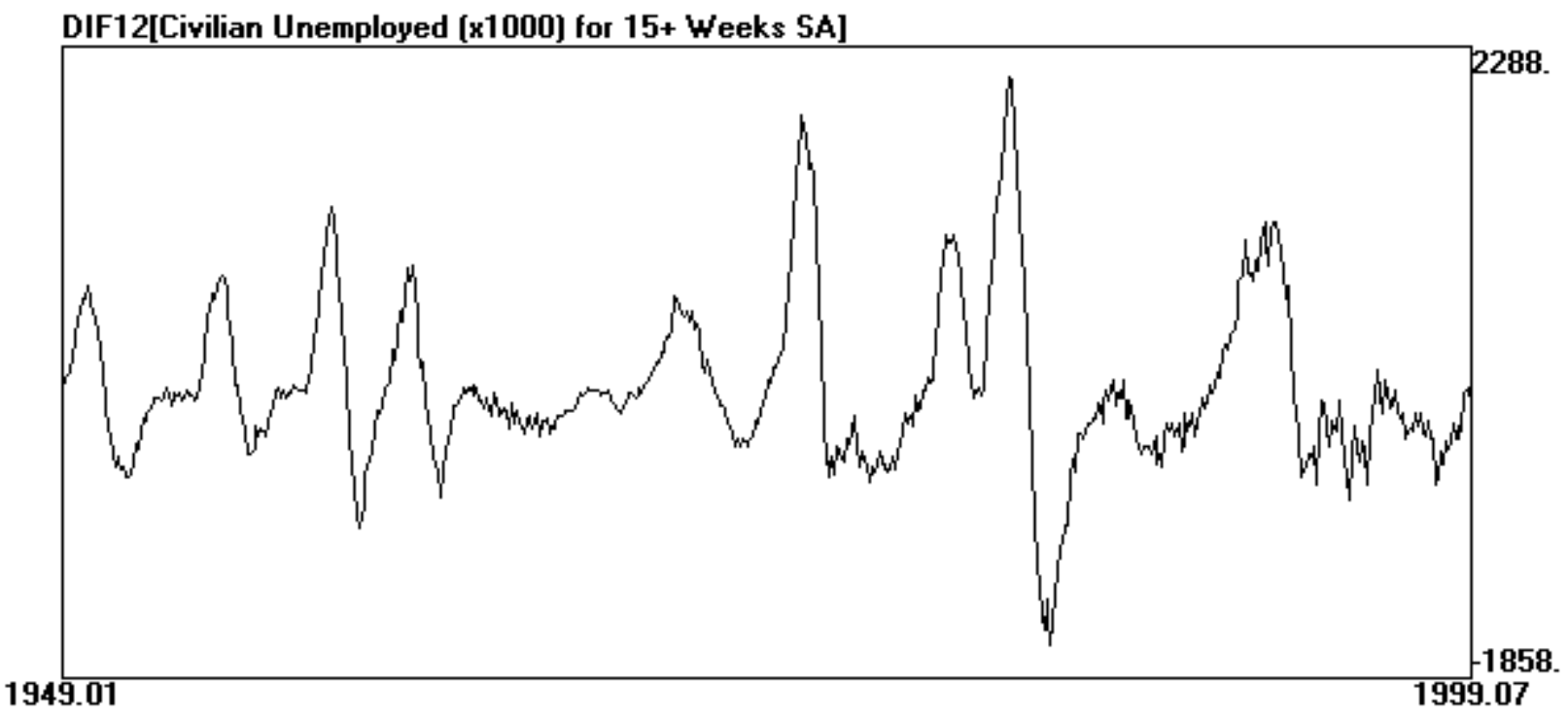

Figure 4: The data

The standardized periodogram 1 (b)) is displayed in Figure 5. The dashed lines are the $90 \%$ and $95 \%$ pointwise con.. dence bands under the stationarity hypothesis, based on (16).

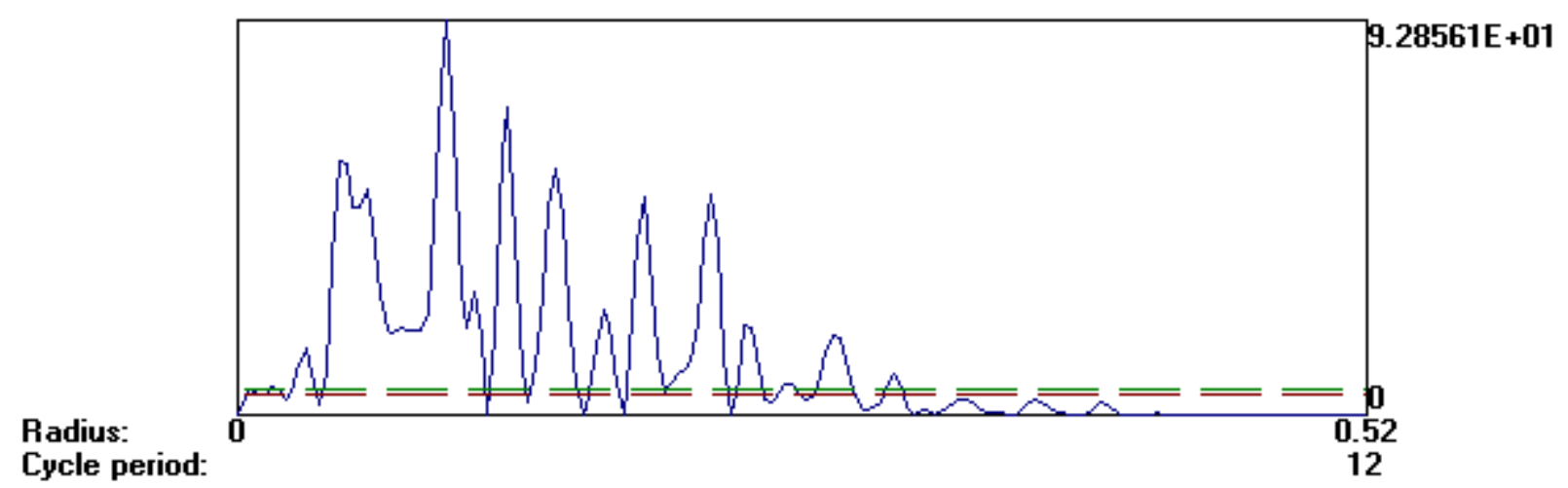


Figure 5: Standardized periodogram b(2)》) of the data

The ..rst peak (with a little dip in the top) corresponds to a cycle duration between 104 and 133 months. The second, and highest, peak corresponds to a cycle of 65 months, and the four next highest peaks correspond to cycles of 50, 43, 33 and 28 months, respectively. These cycle durations are pretty close to the post-W W -II NBER business cycle (trough to trough) durations listed in Diebold and Rudebush (1999, Table 2.1, p.39). The longest postwar NBER cycle duration is 117 month, which corresponds to the little dip in the top of the ..rst peak.

We now test the null hypothesis that this series has 6 pairs of complex conjugate unit roots, with frequencies corresponding to cycles of $117,65,50$, 43, 33 and 28 months:

Tabel 2: Null hypothesis and test results

\begin{tabular}{|c|c|c|c|}
\hline & & & \\
\hline $\begin{array}{l}l_{0}^{0 ; j} \\
0: 05370\end{array}$ & 117 & $\begin{array}{l}0: 07238 \\
0\end{array}$ & \\
\hline 0:09666 & 65 & $0: 13934$ & \\
\hline $0: 12566$ & 50 & $0: 10855$ & \\
\hline $0: 14612$ & 43 & 0:08387 & \\
\hline $0: 19040$ & 33 & $0: 06667$ & \\
\hline $0: 22440$ & 28 & 0:04361 & \\
\hline \multirow{2}{*}{\multicolumn{3}{|c|}{$\begin{array}{l}\text { Test statistic }=\max _{\mathrm{j}} 1, \ldots ; 6,6\left(\mathrm{~A}_{0 ; j}\right)=n= \\
10 \% \text { critical region }= \\
5 \% \text { critical region }= \\
\mathrm{p}_{\mathrm{i}} \text { value } 1 / 4\end{array}$}} & $\begin{array}{l}0: 13934 \\
(0 ; 0: 03366)\end{array}$ \\
\hline & & & $\begin{array}{l}(0 ; 0: 02095) \\
1\end{array}$ \\
\hline
\end{tabular}

Clearly, the complex unit root hypothesis involved is not rejected.

If the time series is actually stationary, then given the speci..ed frequencies

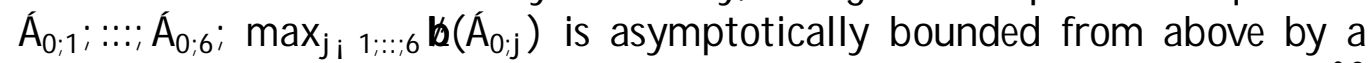
random variable which is distributed as the maximum of 6 independent $\hat{A}_{2}^{2}$ variates. See (26). Dividing the corresponding critical values $\tau_{6}(\circledR)$ [see (27)] by $n$ then yields the critical values of $\left.\max _{j i}{ }_{1 ;: ; 6} \quad A_{0} A_{0 ; j}\right)=n$ under the stationarity hypothesis:

Table 3: Stationarity test results

$\begin{array}{ll}\text { Test statistic }=\max _{\mathrm{j} i 1 ; \cdots ; 6} \quad b\left(\hat{A}_{0 ; j}\right)=n= & 0: 13934 \\ 5 \% \text { critical region }= & (0: 01570 ; 1) \\ 10 \% \text { critical region }= & (0: 01335 ; 1) \\ \mathrm{pi}_{\mathrm{i} \text { value } 1 / 4} & 0\end{array}$


Clearly, the stationarity hypothesis is ..rmly rejected, given the "a priori" chosen frequencies $\hat{A}_{0 ; j} ; j=1 ;: ; 6$; under the null hypothesis.

These results provide evidence that business cycles may indeed be due to complex unit roots. Whether this evidence is compelling depends on how one weighs the pretesting problem mentioned before.

\section{REFERENCES}

Billingsley, P. (1968): Convergence of Probability Measures. New York: J ohn Wiley.

Diebolt, F. X., and G. D. Rudebusch (1999): Business Cycles: Duration, Dynamics, and Forecasting. Princeton: Princeton U niversity Press.

Dudley, R. M. (1968): "Distances of Probability Measures and Random Variables", Annals of Mathematical Statistics, 39, 1563-1572.

Fuller, W. A . (1976): Introduction to Statistical Time Series. New York: J ohn Wiley.

Gaenssler, P. (1983): Empirical Processes. Hayward, California: Institute of $M$ athematical Statistics.

Gregoir, S. (1999a): "Multivariate Time Series with Various Hidden Unit Roots, Part I: Integral Operator A Igebra and Representation Theory", Econometric Theory, 15, 435-468.

Gregoir, S. (1999b): "M ultivariate Time Series with Various Hidden Unit Roots, Part II: Estimation and Testing", E conometric Theory, 15, 469-518.

Gregoir, S. (1999C): "E \& cient Tests for the Presence of a Couple of C omplex Conjugate Unit R oots in Real Time Series", paper presented at the European M eeting of the E conometric Society 1999, Santiago de Compostela, Spain.

Hernndorf, N. (1984): "A Functional Central Limit Theorem for Weakly Dependent Sequences of Random Variables", Annals of Probability, 12, 141153.

Skorohod, A. V. (1956): "Limit Theory for Stochastic Processes", Theory of Probability and its Applications, 1, 261-290.

Wichura, M.J . (1970): "On the Construction of Almost Uniformly Convergent Random Variables with Given Weakly Convergent Image Laws", Annals of Mathematical Statistics, 41, 284-291.

APPENDIX

Proof of (17): First, observe that there exist functions $a(») ; b(») ; c(»)$; and $\mathrm{d}(\gg)$; not depending on $\mathrm{t}$, such that for $\mathrm{t}=1 ; 2 ;:: . ;$ 


$$
\begin{aligned}
& \cos (\gg t)=a(\gg){ }^{Z_{t+1}} \cos (\gg: x) d x+b(\gg){ }^{t+1} \sin (\gg: x) d x ; \\
& \sin (\gg t)=c(\gg) Z_{t}{ }_{t+1}^{t} \cos (\gg: x) d x+d(\gg){ }_{t}^{t+1} \sin (\gg: x) d x:
\end{aligned}
$$

Therefore, it follows from (8) that

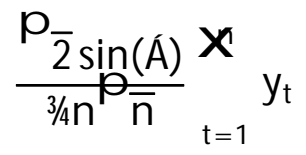

$$
\begin{aligned}
& =\frac{1}{n}{ }_{t=1}^{X^{n}} \cos (A ́ t) W_{1 ; n}(t=n)+\frac{1}{n}{ }_{t=1}^{X^{n}} \sin (A ́ t) W_{2: n}(t=n)+O_{p}\left(1={ }_{1}^{p} \bar{n}\right) \\
& =a(\hat{A}) \quad \cos (n \hat{A}: x) W_{1 ; n}(x) d x+b(») \quad \sin (n \hat{A}: x) W_{1 ; n}(x) d x \\
& Z^{0}{ }_{1} \quad Z_{1}^{0} \\
& c(\hat{A}) \quad \cos (n \hat{A}: x) W_{1 ; n}(x) d x+d(A ́) \quad \sin (n \hat{A}: x) W_{1 ; n}(x) d x \\
& +O_{p}\left(1=\frac{p}{n}\right):
\end{aligned}
$$

Moreover, it is not hard to verify that

$$
E\left[W_{1 ; n}(x) W_{1 ; n}(y)\right]=\min (x ; y)+O(1=n):
$$

Therefore

$$
\begin{aligned}
& E^{\mu Z_{1}} \cos (n A ́ x) W_{1 ; n}(x) d x \\
= & Z_{1} Z_{1}^{0}{ }_{1}^{\prod_{2}} \cos (n A ́ x) \cos (n A ́ y) \min (x ; y) d x d y+O(1=n) \\
= & 0(1=n) ;
\end{aligned}
$$

where the last equality is an elementary calculus result. Thus,

$$
Z_{0} \cos (n A ́ x) W_{1 ; n}(x) d x=O_{p}\left(1=\frac{p}{n}\right) \text { : }
$$

Along the same lines it can be shown that the other terms in (29) are $\mathrm{O}_{\mathrm{p}}(1=\overline{\mathrm{n}})$ : Q.E.D. 
Proof of (18): It follows from (8) that

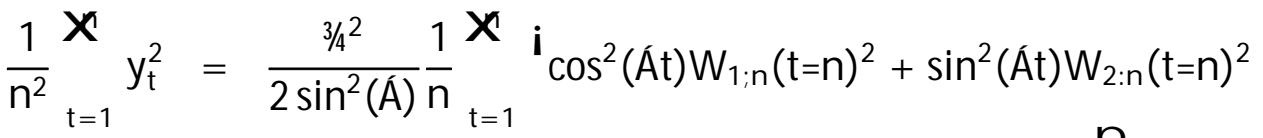

$$
\begin{aligned}
& +2 \cos \left(A ́ \mathrm{t} t \sin (A ́ \mathrm{t}) \mathrm{W}_{1 ; n}(\mathrm{t}=n) \mathrm{W}_{2 ; n}(\mathrm{t}=n)\right)+\mathrm{O}_{\mathrm{p}}\left(1=\mathrm{p}_{\overline{\mathrm{n}}}\right) \\
& =\frac{3 / 4}{4 \sin ^{2}(\dot{A})} \frac{1}{n}_{t=1}^{X_{n}^{n}} W_{1 ; n}(t=n)^{2}+\frac{1}{n}_{t=1}^{X n} W_{2 ; n}(t=n)^{2} \\
& +\frac{1}{n}{ }_{t=1}^{n} \cos (2 \dot{A} t) W_{1 ; n}(t=n)^{2} i \frac{1}{n}_{t=1}^{X n} \cos (2 A ́ t) W_{2 ; n}(t=n)^{2} \\
& +2 \frac{1}{n}_{t=1}^{X^{n}} \sin (2 A ́ t) W_{1 ; n}(t=n) W_{2 ; t}(t=n) \quad:
\end{aligned}
$$

It is easy to show that

$$
\begin{aligned}
& \frac{1}{n}{ }_{t=1}^{X n} W_{1 ; n}(t=n)^{2}=Z_{1} W_{1 ; n}(x)^{2} d x+O_{p}(1=n) ; \\
& \frac{1}{n}{ }_{t=1}^{n} W_{2 ; n}(t=n)^{2}={ }_{0}^{1} W_{2 ; n}(x)^{2} d x+O_{p}(1=n) ;
\end{aligned}
$$

hence by the continuous mapping theorem [see Billingsley (1968)],

$$
\left.\frac{1}{n}{ }_{t=1}^{X^{n}} W_{1 ; n}(t=n)^{2}+\frac{1}{n}{ }_{t=1}^{X^{n}} W_{2 ; n}(t=n)^{2}\right){ }_{0}^{Z_{1}} W_{1}(x)^{2} d x+\sum_{0}^{Z_{1}} W_{2}(x)^{2} d x:
$$

Moreover, it follows similarly to (29) that

$$
\begin{aligned}
& \frac{1}{n}_{t=1}^{X n} \cos (2 \dot{A} t) W_{1 ; n}(t=n)^{2} \\
= & a(2 A ́) Z_{1} \cos (2 n A ́ x) W_{1 ; n}(x)^{2} d x \\
& +b(2 \dot{A}) Z_{0}^{1} \sin (2 n A ́ x) W_{1 ; n}(x)^{2} d x+O_{p}(1=n) ;
\end{aligned}
$$




$$
\begin{aligned}
& \frac{1}{n}{ }_{t=1}^{X} \sin (2 A ́ t) W_{1 ; n}(t=n) W_{2 ; t}(t=n) \\
= & c(2 A ́) Z_{1} \cos (2 n A ́ x) W_{1 ; n}(x) W_{2 ; n}(x) d x \\
& +d(2 A ́){ }_{0}^{0} \sin (2 n A ́ x) W_{1 ; n}(x) W_{2 ; n}(x) d x+O_{p}(1=n):
\end{aligned}
$$

In analyzing the asymptotic properties of continuous functions of $W_{1 ; n}$ and/ or $W_{2 ; n}$, it often suф ces to analyze the properties of the same functions of the independent standard $W$ iener processes $W_{1} ; W_{2}$; because of the Skorohod (1956), Dudley (1968), and W ichura (1970) representation theorem. See also Gaenssler, P. (1983, p. 83). Loosely speaking, this representation theorem states that there exist versions $\bar{W}_{n}=\left(\bar{W}_{1 ; n} ; \bar{W}_{2 ; n}\right)^{0}$ and $\bar{W}=\left(\bar{W}_{1} ; \bar{W}_{2}\right)^{0}$ of $W_{n}=\left(W_{1 ; n} ; W_{2 ; n}\right)^{0}$ and $W=\left(W_{1} ; W_{2}\right)^{0}$, respectively, such that $W_{n}$ has the same distribution as $W_{n} ; \bar{W}$ has the same distribution as $W$ (namely a bivariate standard $W$ iener process), and $\bar{W}_{n} ! \bar{W}_{\text {a.s. }}{ }^{6}$

Due to the representation theorem, the limiting distribution of

$$
Z_{0}^{Z_{1}} \cos (2 n A ́ x) W_{1 ; n}(x)^{2} d x
$$

is the same as the limiting distribution of

$$
Z_{1} \cos (2 n A ́ x) W_{1}(x)^{2} d x:
$$

The latter limited distribution is constant zero, because.

$$
\begin{aligned}
& \mu Z_{1} \\
& \text { E } \cos (2 n A ́ x) W_{1}(x)^{2} d x \\
& =\underbrace{Z_{1} Z_{1}^{0}}_{0} \cos (2 n A ́ x) \cos (2 n A ́ y) E{ }^{f} W_{1}(x)^{2} W_{1}(y)^{2^{\alpha a}} d x d y
\end{aligned}
$$

${ }^{6} \mathrm{M}$ ore precisely,

$$
P \lim _{n ! 1} 1 / 2 \bar{W}_{n} ; \bar{W}^{\dot{d}}=1
$$

where $1 / 2$ is the Skorohod norm on the space $D^{2}[0 ; 1]$ of right-continuous mappings from $[0 ; 1]$ into $R^{2}$. See Billingsley (1968). 


$$
\begin{aligned}
& =Z_{1} Z_{1} \cos (2 n A ́ x) \cos (2 n A ́ y){ }^{i} 2(\min (x ; y))^{2}+x y^{\phi} d x d y \\
& =0(1=n):
\end{aligned}
$$

The second equality is a standard Wiener measure calculus result, and the last equality is an easy calculus exercise. Thus by Chebishev's inequality

$$
Z_{1} \cos (2 n A ́ x) W_{1 ; n}(x)^{2} d x ! 0 \text { : }
$$

The same applies to the sinus case. Along the same lines it can be shown that

$$
Z_{0} \cos (2 n A ́ x) W_{1 ; n}(x) W_{2 ; n}(x) d x ! 0 \text {; }
$$

and the same applies to the sinus case. Q.E.D.

Proof of (19): It follows from (8) that

$$
\begin{aligned}
& \frac{1}{n}_{\bar{P}}^{\frac{1}{n}}{ }_{t=1}^{n} y_{t} \cos (A ́ t)=\frac{3 / 4}{\overline{2} \sin (\dot{A})} \frac{1}{n}{ }_{t=1}^{X_{n}^{n}} \cos ^{2}(A ́ t) W_{1 ; n}(t=n) \\
& +P_{\overline{2}} \sin (\dot{A}) \frac{1}{n}{ }_{t=1}^{X n} \cos (A ́ t) \sin (A ́ t) W_{2 ; n}(t=n) \\
& =\frac{2}{2}^{p} \overline{2}^{3 / 4} \sin (\dot{A}) \frac{1}{n}_{t=1}^{X n} W_{1 ; n}(t=n) \\
& +\frac{p^{p}}{2} \overline{2} \sin (\dot{A}) \frac{1}{n}_{t=1}^{X^{n}} \cos (2 \dot{A} t) W_{1 ; n}(t=n)
\end{aligned}
$$

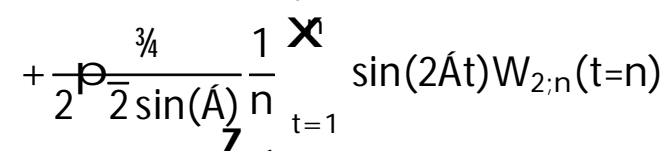

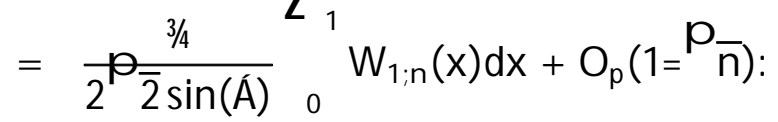

The last step follows similarly to the proof of (17). Similarly,

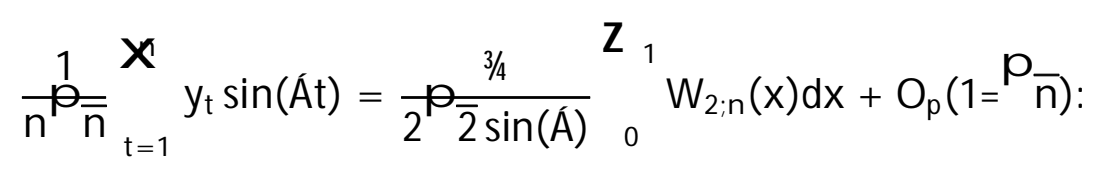


Part (19) of Lemma 5 follows now from the continuous mapping theorem. Q.E.D.

Proof of (20): Similarly to the proof of (19). Q.E.D.

Proof of Theorem 2: We only need to prove the parts (22) and (23), because the other results in Theorem 2 follow straightforwardly from Lemma 5 and its proof.

Denote

$$
\begin{aligned}
& a_{m}=Z_{Z_{1}^{0}}^{\mu Z_{1}} W_{1 ; m}(x) d x+\mathbb{1}_{2}+\mu Z_{1} W_{2 ; m}(x) d x^{\mathfrak{I}_{2}} ; \\
& b_{m}={ }_{0} W_{1 ; m}(x)^{2} d x+{ }_{0} W_{2 ; m}(x)^{2} d x
\end{aligned}
$$

Then

$$
\tilde{A}_{k}\left(\hat{A}_{j}\right)=\frac{P_{\substack{k \\ m=1}}^{2} a_{j}}{{ }_{m}^{2} b_{m}} ;
$$

hence for $\mathrm{j}=1 ; 2 ;: ; \mathrm{k}$;

$$
!_{\mathrm{j}}^{2}=\frac{!_{1}^{2} \mathrm{a}_{1} \tilde{\mathrm{A}}_{\mathrm{k}}\left(\hat{\mathrm{A}}_{\mathrm{j}}\right)}{\mathrm{a}_{\mathrm{j}} \tilde{\mathrm{A}}_{\mathrm{k}}\left(\hat{\mathrm{A}}_{1}\right)}:
$$

Substituting (36) in (36) yields

$$
\mathrm{X}_{\mathrm{m}=1}^{\mathrm{K}} \tilde{\mathrm{A}}_{k}\left(\dot{A}_{\mathrm{m}}\right) \frac{\mathrm{b}_{\mathrm{m}}}{\mathrm{a}_{\mathrm{m}}}=1 ;
$$

hence

$$
\min _{m=1 ;: ; ; k} \tilde{A}_{k}\left(\dot{A}_{m}\right) X_{m=1}^{X^{k}} \frac{b_{m}}{a_{m}} \cdot 1 \cdot \max _{m=1 ;: ; ; k} \tilde{A}_{k}\left(\dot{A}_{m}\right){ }_{m=1}^{X^{k}} \frac{b_{m}}{a_{m}}:
$$

Q.E.D. 